\title{
A gas chromatography-mass spectrometry-based metabolomic approach for the characterization of goat milk compared with cow milk
}

\author{
Paola Scano, ${ }^{*}$ Antonio Murgia, ${ }^{*}$ Filippo M. Pirisi, $\dagger$ and Pierluigi Caboni $\dagger^{1}$ \\ *Department of Chemical and Geological Sciences, and \\ †Department of Life and Environmental Sciences, University of Cagliari, 72-09124 Cagliari, Italy
}

\begin{abstract}
In this work, the polar metabolite pool of commercial caprine milk was studied by gas chromatography-mass spectrometry and multivariate statistical data analysis. Experimental data were compared with those of cow milk and the discriminant analysis correctly classified milk. By the same means, differences due to heat treatments (UHT or pasteurization) on milk samples were also investigated. Results of the 2 discriminant analyses were combined, with the aim of finding the discriminant metabolites unique for each class and shared by 2 classes. Valine and glycine were specific to goat milk, talose and malic acid to cow milk, and hydroxyglutaric acid to pasteurized samples. Glucose and fructose were shared by cow milk and UHT-treated samples, whereas ribose was shared by pasteurized and goat milk. Other discriminant variables were not attributed to specific metabolites. Furthermore, with the aim to reduce food fraud, the issue of adulteration of caprine milk by addition of cheaper bovine milk has been also addressed. To this goal, mixtures of goat and cow milk were prepared by adding the latter in a range from 0 to $100 \%$ (vol/vol) and studied by multivariate regression analysis. The error in the level of cow milk detectable was approximately $5 \%$. These overall results demonstrated that, through the combined approach of gas chromatography-mass spectrometry and multivariate statistical data analysis, we were able to discriminate between milk typologies on the basis of their polar metabolite profiles and to propose a new analytical method to easily discover food fraud and to protect goat milk uniqueness. The use of appropriate visualization tools improved the interpretation of multivariate model results.
\end{abstract}

Key words: goat milk, gas chromatography-mass spectrometry, metabolomics, heat treatment, food fraud

Received April 16, 2014

Accepted June 26, 2014.

${ }^{1}$ Corresponding author: caboni@unica.it

\section{INTRODUCTION}

Goat (Capra hircus) milk and related dairy products have nowadays gained a valuable industry niche (Dubeuf et al., 2004). Many commercial parameters indicate that the diffusion of goat dairy products is increasing as whole milk, fermented milk derivatives, dried or evaporated milk, and for the production of cheese. The nutritional and health benefits of goat milk are of the utmost relevance for people affected by food allergies, with bovine milk proteins the dominant food cause. Although controversial, superior digestibility of goat milk compared with cow milk, attributed to the higher content of the $\alpha_{\mathrm{s} 2}-\mathrm{CN}$ variant rather than $\alpha_{\mathrm{s} 1^{-}}$ $\mathrm{CN}$, lower naturally homogenized fat globule size, and the higher proportion of medium-chain triacylglycerols has been commonly accepted. Despite the huge number of reports on its nutraceutical properties (Silanikove et al., 2010; Ceballos et al., 2009), the use of goat milk for nutraceutical needs still deserves in-depth discussion and documentation (Haenlein, 2004). Milk is a very complex mixture of several components in different physical states. Milk composition is influenced by a range of different factors (e.g., diet, genetics, number and stage of lactation, seasonal variation, SCC, and milk processing; Goetsch et al., 2011). These factors may have remarkable quantitative effects on milk nutrients as well as on the physical and technological properties of milk (e.g., coagulation properties, heat stability, and fermentation quality of the milk; Dubeuf et al., 2004). Whereas lipids and lactose are the 2 major caloric nutrients, milk also contains a wide variety of bioactive compounds, including immunoglobulins and other immune proteins, peptides, nucleotides, oligosaccharides, and metabolites (Raynal-Ljutovac et al., 2008; Sundekilde et al., 2013). Sugars, free amino acids, organic acids, and other lowmolecular-weight compounds compose the metabolite pool of milk. The different origin and sources of these compounds contribute to the variability of milk metabolite profiles. Milk metabolites often reflect metabolic activity in the mammary gland or metabolism in the whole organism, or both; they may also originate from enzymatic reactions or from microorganisms present in 
raw milk, or both (Sundekilde et al., 2013). Moreover, before entering the market, milks undergo different heat treatments that determine their commercial value and their quality; these treatments can modify the overall metabolite composition of milk. It was found that levels of monosaccharides in milk change because of thermal processing (Mendoza et al., 2005) and during storage (Troyano et al., 1996). The characterization of the metabolite profile in a biological matrix is well performed by metabolomics; this science is based on the use of analytical methods, such as GC-MS (Marincola et al., 2012), nuclear magnetic resonance (Locci et al., 2011), and direct analysis in real time-mass spectrometry (Hrbek et al., 2014), coupled with multivariate statistical data analysis (MVA). Metabolomic studies have been extensively applied in the areas of nutrition sciences and food matrices, such as milk (Chen et al., 2004; Boudonck et al., 2009; Klein et al., 2010; Marincola et al., 2012; Harzia et al., 2013; Sundekilde et al., 2013; Hrbek et al., 2014).

The risk linked to food fraud is increasing due to the global and composite nature of food supply chains. With the aim to reduce this risk and in view of detecting economically driven adulterations, in this study, the issue of adulteration of caprine milk by the addition of cheaper bovine milk was addressed. The commercial value of goat milk is much higher than that of cow milk due to lower productivity and little market demand; therefore, the addition of cow milk to goat milk can allow economic advantages and becomes a fraud when the mixture is sold with label. Taking into account this option, in this paper, an attempt to assess whether the metabolomic approach could be suitable tool for discovering such fraud was carried out. The literature reports several attempts to find suitable methods to detect milk adulteration. Quantification of cow milk adulteration of goat milk, based on solvent separation of whey proteins, followed by HPLC with electrospray ionization mass spectrometry, was performed by Chen et al. (2004); levels as low as $5 \%$ of cow milk were detected. Levieux and Venien (1994) proposed an ELISA to detect cow $\beta$-LG at $5 \mathrm{ng} / \mathrm{mL}$. Antonilli et al. (2005), by inspection of the ratios of some FAME, offered some parameters suitable for discovering such fraud. Proton nuclear magnetic resonance low-molecular-weight metabolite fingerprinting was applied for the quantification of the relative amount of cow and sheep milk in mixtures (Lamanna et al., 2011).

In this work, for the first time, the goat milk metabolite profile, composed by polar and hydrophilic low-molecular-weight compounds, was characterized by the means of GC-MS and compared, through the application of discriminant multivariate analysis, with cow milk. Differences in milk metabolite profiles correlated with heat treatments: UHT and pasteurization were also investigated. Moreover, milk mixtures of goat milk with increasing quantities of cow milk were prepared and their GC-MS metabolic profiles used to construct a suitable model, based on orthogonal projections to latent structures (OPLS) regression, to detect adulteration of goat milk with cow milk.

\section{MATERIALS AND METHODS}

\section{Chemicals and Reagents}

Methanol, chloroform, hexane, pyridine, methoxamine hydrochloride, potassium chloride, $N$-methyl- $N$ (trimethylsilyl)trifluoroacetamide, lactic acid, valine, butyric acid, urea, glycine, succinic acid, fumaric acid, serine, malic acid, proline, alanine, creatinine, glutamine, phosphoric acid, fructose, glucose, galactose, gluconic acid, palmitic acid, inositol and stearic acid were purchased from Sigma-Aldrich (Milan, Italy). Bidistilled water was obtained from a Milli-Q purification system (Millipore S.p.A., Milan, Italy) before use.

\section{Samples}

Seventeen commercial samples of goat whole milk (G1-G17) and 14 samples of cow whole milk (C1C14) were acquired in local markets; all samples were within the expiration date. Seventeen milk samples were subjected to UHT and 14 samples were subjected to pasteurization processes. Furthermore, 9 mixtures were prepared by adding different aliquots $(\%, \mathrm{vol} / \mathrm{vol})$ of cow milk to goat milk as follows: $0,5,10,20,40,50$, 60,80 , and $100 \%$.

\section{Extraction and Derivatization}

To obtain rupture of the milk micelles, $15 \mathrm{~mL}$ of sample was sonicated for $15 \mathrm{~min} ; 100 \mu \mathrm{L}$ of milk was transferred to an Eppendorf tube and then $250 \mu \mathrm{L}$ of methanol and $125 \mu \mathrm{L}$ of chloroform were added. Samples were vortexed every 15 min 4 times and then $380 \mu \mathrm{L}$ of chloroform and $90 \mu \mathrm{L}$ of aqueous $0.2 \mathrm{M}$ potassium chloride were added. The suspension was centrifuged at $13,572 \times g$ for $10 \mathrm{~min}$ at $4^{\circ} \mathrm{C}$. After centrifugation, the aqueous layer was transferred to a glass vial and dried by a gentle nitrogen stream and derivatized with $50 \mu \mathrm{L}$ of pyridine containing methoxamine hydrochloride at $10 \mathrm{mg} / \mathrm{mL}$. After $17 \mathrm{~h}, 100 \mu \mathrm{L}$ of $N$-methyl- $N$ (trimethylsilyl)trifluoroacetamide was added and after $1 \mathrm{~h}$, samples were resuspended with $600 \mu \mathrm{L}$ of hexane. 


\section{GC-MS Analysis}

One microliter of derivatized samples was injected splitless into a 6850 gas chromatograph coupled with a 5973 Network mass spectrometer (Agilent Technologies Inc., Santa Clara, CA). The injector temperature was $200^{\circ} \mathrm{C}$. The gas flow rate through the column was $1 \mathrm{~mL} / \mathrm{min}$. The fused silica capillary column was a 0.25- $\mu \mathrm{m}$ DB5-MS column $(30 \mathrm{~m} \times 0.25 \mathrm{~mm}$ i.d.; J\&W Scientific Inc., Folsom, CA). The initial temperature program was as follows: 3 min of isothermal heating at $50^{\circ} \mathrm{C}$, which was then increased to 250 at $3^{\circ} \mathrm{C} / \mathrm{min}$ and held at $250^{\circ} \mathrm{C}$ for $25 \mathrm{~min}$. The transfer line and the ion source temperatures were 280 and $180^{\circ} \mathrm{C}$, respectively. Ions were generated at $70 \mathrm{eV}$ with electron ionization and were recorded at $1.6 \mathrm{scans} / \mathrm{s}$ over the mass range $m / z 50$ to 550 . The GC-MS data analysis was conducted by integrating each resolved chromatogram peak. Identification of metabolites was performed using the standard NIST08 mass spectra library (http://www. nist.gov/srd/mslist.cfm), a library developed at the Max Planck Institute of Golm (Germany), and, when available, by comparison with authentic standards.

\section{Multivariate Statistical Data Analysis}

A $31 \times 40$ matrix composed of the analyzed milk samples (31 samples) and the chromatographic peak areas (40 variables) was constructed together with a $\mathbf{Y}$ matrix with sample information: milk typologies (goat and cow) and heat treatments (pasteurization and UHT). In addition, a second $\mathbf{X}$ matrix $(9 \times 41)$ was constructed; it was composed of the 9 mixtures, the 40 $\mathbf{x}$ variables and 1 continuous $\mathbf{y}$ variable consisting of the percentages of cow milk added. For both of them, the median fold change normalization row-wise was selected for adjusting peak intensities between samples to a common scale. This centering method assumes that measured peak intensities are directly proportional to concentrations of metabolites in solution. Under this assumption, the change in intensity of a profile due to variable sample dilution or inconsistency of GC-MS acquisition parameters, or both, are expected to be uniform across all peaks and thus a fixed scaling factor is used (Veselkov et al., 2011). When a variable presented skew distribution it was logarithmically transformed and the improvement of the symmetry evaluated using the skewness test statistics as implemented in SIMCA-P software (version 13.0; MKS Umetrics AB, Umeå, Sweden). Prior to analysis, each data matrix was mean centered and unit variance scaled columnwise. The obtained matrices were submitted to MVA. Principal components analysis (PCA), the partial least squares (PLS) method, PLS-discriminant analysis
(PLS-DA), and their orthogonal extensions (OPLS and OPLS-DA; Eriksson et al., 2013) were performed with SIMCA-P software. The quality of the models was evaluated on the basis of the cumulative parameters $R^{2} X$ (variation in $\mathbf{X}$ explained by the model), $R^{2} Y$ (amount of $\mathbf{Y}$ explained by the model), and $\mathrm{Q}^{2}$ (i.e., the cumulated cross-validation for $\mathrm{R}^{2} \mathrm{Y}$; or $\mathrm{R}^{2} \mathrm{X}$ for the $\mathrm{PCA}$ ), estimated by the default leave-one-seventh-out cross-validation in the corresponding PLS-DA model. Models were tested for overfitting using the y-table permutation test $(\mathrm{n}=400)$ as implemented in the SIMCA-P+ program. Jackknifed standard errors were calculated from all rounds of cross-validation. Accuracy for the PLS model, in which the leave-one-out crossvalidation was performed, was evaluated by the root mean square error in cross-validation (Eriksson et al., 2013). Results of the OPLS-DA models were also compared and reported as a shared and unique structures (SUS) plot; this is a 2-dimensional scatter plot of the loading correlation vectors of the predictive components of 2 separate models (Wiklund et al., 2008).

\section{RESULTS AND DISCUSSION}

A total of 31 commercial samples of goat and cow whole milks were studied by GC-MS; from the analysis of the chromatograms, we selected a total of 40 polar metabolites reported in Table 1. Twelve compounds were not identified and they will be hereafter named U1 to U12. The chemical composition analysis revealed that the aqueous fraction obtained by the extraction procedure was rich in short-chain hydroxylated carboxylic acids, such as lactic acid, succinic acid, fumaric acid, malic acid, 2-hydroxyglutaric acid, and gluconic acid; long-chain stearic and palmitic acids were also found. Among free amino acids, serine, valine, glycine, alanine, proline, and glutamine were detected. Also the saccharides D-glucose, fructose, talose, inositol, and galactose were identified. In Table 2, we reported the normalized areas of each metabolite as means and standard deviations over all samples for the 2 typologies of milk. In this work, aimed at observing also the variability of the detected metabolites within each milk typology, we also reported the coefficient of variation $(\mathrm{SD} /$ mean). The reported coefficient of variation for each variable gives a measure of the dispersion of the variable among samples in a way that does not depend on the variable measurement unit; this allowed us to directly compare the coefficients of variation, higher values of which indicated a greater dispersion in the variable. By the Student's $t$-test, we also tested the null hypothesis (the means are not significantly different among the 2 sets of samples) and we reported the results in Table 2. In this table, we observed that, with 
a high level of confidence $(P<0.01)$, the null hypothesis was rejected for a great number of metabolites $(27$ out of 40). The sum of coefficient of variation values was 27.8 and 18 for goat and cow milk samples, respectively, indicating a greater variability of the metabolite concentrations within caprine milk. The data we reported in Table 2 were the results of univariate tests that analyze each variable separately. Conversely, in biological samples, the variables are often interconnected and only a multivariate approach is able to fully describe these systems, taking into consideration several variables and their relationship simultaneously. Under this perspective, we constructed a matrix composed of the normalized area of chromatographic data for the analyzed samples and we submitted it to MVA. Initially, for sample distribution overview, to detect outliers, deviating features, and common trends, we performed a PCA; the first 2 principal components accounted for $45 \%$ of the total variance. We report the results in the score plot, shown in the top panel of Figure 1; here, we observed that samples of cow and goat milk clustered in different areas of the plot and goat samples were very scattered. We concluded that cow milk samples had similar characteristics that differed from those of goat milk, and that goat milk, based on the detected variables, had a more heterogeneous composition, as already observed from the analysis of results in Table 2. Based on Hotelling's $\mathrm{T}^{2}$ test at $99 \%$ confidence, sample G12 was identified as an outlier, and because no evident reasons for its deviating features were found and, moreover, its behavior was in trend with sample G15, we decided to keep this sample

Table 1. The GC-MS characteristics of milk metabolites

\begin{tabular}{|c|c|c|c|c|}
\hline Compound & $\begin{array}{l}\text { Retention } \\
\text { time (min) }\end{array}$ & $\begin{array}{c}\text { EI-MS }{ }^{1}[m / z(\mathrm{amu}) \\
\text { with relative abundance } \\
(\%) \text { in parentheses }]\end{array}$ & Trivial name & Abbreviation \\
\hline 2-Hydroxypropanoic acid & 9,352 & $147(100), 73(80), 117(74)$ & Lactic acid & Lac \\
\hline Valine & 9,713 & $72(100), 75(31), 73(27)$ & Valine & Val \\
\hline Butanoic acid & 10,879 & $147(100), 117(52), 73(53)$ & Butyric acid & $\mathrm{BA}$ \\
\hline Unknown 1 & 11,665 & $73(100), 228(90), 184(39)$ & & U1 \\
\hline Urea & 12,139 & $147(100), 189(62), 171(58)$ & Urea & Urea \\
\hline Unknown 2 & 12,533 & $158(100), 73(64), 159(15)$ & & $\mathrm{U} 2$ \\
\hline Glycine & 13,02 & $174(100), 73(34), 248(18)$ & Glycine & Gly \\
\hline Butanedioic acid & 13,064 & $147(100), 73(41), 75(17)$ & Succinic acid & Suc \\
\hline 2-Butenedioic acid & 13,495 & $245(100), 147(40), 73(37)$ & Fumaric acid & Fum \\
\hline Serine & 13,782 & $204(100), 73(63), 218(55)$ & Serine & Ser \\
\hline Unknown 3 & 14,610 & $73(100), 174(74), 248(58)$ & & U3 \\
\hline Hydroxybutanedioic acid & 15,430 & $73(100), 74(86), 147(74)$ & Malic acid & Mal \\
\hline L-Proline & 15,820 & $156(100), 73(55), 147(20)$ & Proline & Pro \\
\hline Alanine & 15,883 & $84(100), 174(86), 75(47)$ & Alanine & Ala \\
\hline Creatinine & 16,279 & $115(100), 73(67), 329(33)$ & Creatinine & Crn \\
\hline 2,3,4-Trihydroxybutyric acid & 16,399 & $73(100), 147(65), 292(43)$ & 2,3,4-Trihydroxybutyric acid & 3-HBA \\
\hline 2-Hydroxypentanedioic & 16,470 & $73(100), 147(49), 129(41)$ & Hydroxyglutaric acid & HGA \\
\hline Glutamine & 16,992 & $246(100), 73(46), 128(21)$ & Glutamine & Gln \\
\hline Unknown 4 & 18,366 & $117(100), 73(54), 160(14)$ & & $\mathrm{U} 4$ \\
\hline Unknown 5 & 18,419 & $254(100), 357(51), 73(45)$ & & U5 \\
\hline Unknown 6 & 18,497 & $117(100), 73(71), 147(25)$ & & U6 \\
\hline Glycerophosphoric acid & 18,691 & $357(100), 299(95), 73(72)$ & Phosphoglycerate & $\mathrm{PG}$ \\
\hline Unknown 7 & 18,793 & $73(100), 292(69), 147(46)$ & & $\mathrm{U} 7$ \\
\hline Phosphoric acid & 18,884 & $292(100), 174(28), 217(20)$ & Phosphoric acid & $\mathrm{PA}$ \\
\hline Unknown 8 & 19,422 & $73(100), 147(51), 117(23)$ & & U8 \\
\hline Unknown 9 & 19,489 & $73(100), 217(60), 147(40)$ & & U9 \\
\hline D-Fructose & 19,929 & $73(100), 103(68), 217(51)$ & Fructose & Frc \\
\hline Unknown 10 & 19,990 & $73(100), 331(54), 147(33)$ & & U10 \\
\hline Unknown 11 & 20,144 & $204(100), 73(50), 205(20)$ & & U11 \\
\hline D-Glucose & 20,203 & $73(100), 319(80), 205(61)$ & Glucose & Glc \\
\hline D-Galactose & 20,601 & $73(100), 333(87), 292(59)$ & Galactose & Gal \\
\hline Talose & 20,955 & $204(100), 73(52), 191(46)$ & Talose & Tal \\
\hline D-Gluconic acid & 21,243 & $73(100), 147(47), 333(40)$ & Gluconic acid & $\mathrm{GcA}$ \\
\hline Hexadecanoic acid & 21,29 & $73(100), 117(75), 313(73)$ & Palmitic acid & $\mathrm{PmA}$ \\
\hline Inositol & 22,050 & $73(100), 217(72), 305(72)$ & Inositol & Ino \\
\hline D-Ribose & 22,240 & $73(100), 315(44), 299(34)$ & Ribose & Rib \\
\hline D-Mannitol & 22,553 & $73(100), 319(70), 205(50)$ & Mannitol & Man \\
\hline Octadecanoic acid & 23,077 & $73(100), 341(51), 117(49)$ & Stearic acid & StA \\
\hline Unknown 12 & 24,182 & $73(100), 315(82), 217(45)$ & & U12 \\
\hline Unknown 13 & 24,317 & $73(100), 387(82), 299(54)$ & & U13 \\
\hline
\end{tabular}

${ }^{1}$ Electron impact-mass spectrometry. 
for further analysis. The role of the variables in sample distribution can be evinced by the analysis of the loading plot shown in the bottom panel of Figure 1. Here, considering that samples clustered based on their animal provenience along the first principal component, we assumed that variables in the right side of the plot (ribose, glycine, and U9, among others) characterized goat milk and sugars (glucose, fructose, and talose) characterized cow milk. The deviating samples, G12 and G15, were high in succinate, a metabolite entering the Krebs cycle. To classify goat and cow milk based on their metabolite profiles, we performed an OPLS-DA. This discriminant analysis, with a high degree of confidence, correctly classified samples and well separated the 2 classes of milk, producing a model with $\mathrm{R}^{2}(\mathrm{X})=$ $0.43, \mathrm{R}^{2}(\mathrm{Y})=0.95$, and $\mathrm{Q}^{2}(\mathrm{Y})=0.87$, with 1 predictive component and 1 orthogonal component; the resulting score plot is shown in the top panel of Figure 2. The analysis of loading values along the predictive component, (bottom panel of Figure 2), indicated that the most important variables in discriminating the 2 classes [i.e., those having the largest values (negative or positive)] were glycine, ribose, and valine for goat milk (positive values in the bottom panel of Figure 2), and U5, U11, glucose, and talose for cow milk. In the orthogonal direction, interclass variability was dominated by the difference of the metabolite profiles due to the heat treatments. Consequently, we also compared UHT

Table 2. Metabolite composition (peak area, \%), as calculated by GC-MS, of goat and cow milk ${ }^{1}$

\begin{tabular}{|c|c|c|c|c|c|c|c|}
\hline \multirow[b]{2}{*}{ Compound } & \multicolumn{3}{|c|}{ Goat $(\mathrm{n}=17)$} & \multicolumn{3}{|c|}{$\operatorname{Cow}(\mathrm{n}=14)$} & \multirow[b]{2}{*}{$P$-value ${ }^{2}$} \\
\hline & Mean & $\mathrm{SD}$ & $\mathrm{CV}$ & Mean & $\mathrm{SD}$ & $\mathrm{CV}$ & \\
\hline Lactic acid & 5.47 & 4.75 & 0.87 & 1.79 & 0.62 & 0.35 & $0.007^{* *}$ \\
\hline Valine & 0.56 & 0.47 & 0.84 & 0.10 & 0.04 & 0.37 & $0.001^{* *}$ \\
\hline Butyric acid & 0.20 & 0.11 & 0.56 & 0.33 & 0.13 & 0.38 & $0.004^{* *}$ \\
\hline Unknown 1 & 0.84 & 0.26 & 0.31 & 0.55 & 0.23 & 0.41 & $0.004^{* *}$ \\
\hline Urea & 62.50 & 10.42 & 0.17 & 49.15 & 6.09 & 0.12 & $0.000^{* *}$ \\
\hline Unknown 2 & 0.25 & 0.40 & 1.63 & 0.08 & 0.10 & 1.26 & 0.13 \\
\hline Glycine & 4.99 & 2.40 & 0.48 & 1.20 & 0.24 & 0.20 & $0.000^{* *}$ \\
\hline Succinic acid & 1.34 & 2.54 & 1.89 & 0.31 & 0.14 & 0.46 & 0.14 \\
\hline Fumaric acid & 0.15 & 0.16 & 1.07 & 0.23 & 0.08 & 0.32 & 0.081 \\
\hline Serine & 0.09 & 0.10 & 1.12 & 0.05 & 0.03 & 0.56 & 0.10 \\
\hline Unknown 3 & 0.04 & 0.01 & 0.32 & 0.02 & 0.01 & 0.30 & $0.000^{* *}$ \\
\hline Malic acid & 0.24 & 0.30 & 1.23 & 0.66 & 0.20 & 0.30 & $0.000^{* *}$ \\
\hline Proline & 0.70 & 0.37 & 0.52 & 0.73 & 0.21 & 0.29 & 0.80 \\
\hline Alanine & 0.15 & 0.10 & 0.63 & 0.38 & 0.31 & 0.80 & $0.007 * *$ \\
\hline Creatinine & 1.04 & 0.63 & 0.60 & 0.68 & 0.27 & 0.40 & 0.054 \\
\hline 2,3,4-Trihydroxybutyric acid & 0.14 & 0.11 & 0.78 & 0.09 & 0.05 & 0.49 & 0.16 \\
\hline Hydroxyglutaric acid & 2.28 & 1.00 & 0.44 & 1.34 & 0.52 & 0.39 & $0.003^{* *}$ \\
\hline Glutamine & 1.86 & 0.89 & 0.48 & 3.05 & 0.65 & 0.21 & $0.000^{* *}$ \\
\hline Unknown 4 & 0.85 & 0.61 & 0.71 & 0.88 & 0.23 & 0.26 & 0.86 \\
\hline Unknown 5 & 1.21 & 0.44 & 0.36 & 8.65 & 2.04 & 0.24 & $0.000^{* *}$ \\
\hline Unknown 6 & 0.27 & 0.22 & 0.80 & 0.25 & 0.05 & 0.20 & 0.77 \\
\hline Phosphoglycerate & 2.16 & 1.31 & 0.61 & 3.17 & 2.02 & 0.64 & 0.10 \\
\hline Unknown 7 & 0.13 & 0.11 & 0.88 & 0.23 & 0.10 & 0.45 & $0.015^{* *}$ \\
\hline Phosphoric acid & 0.24 & 0.12 & 0.49 & 0.20 & 0.26 & 1.32 & 0.52 \\
\hline Unknown 8 & 0.54 & 0.21 & 0.39 & 1.70 & 0.62 & 0.37 & $0.000 * *$ \\
\hline Unknown 9 & 4.81 & 1.28 & 0.27 & 2.78 & 0.64 & 0.23 & $0.000 * *$ \\
\hline Fructose & 0.09 & 0.08 & 0.87 & 0.21 & 0.04 & 0.22 & $0.000^{* *}$ \\
\hline Unknown 10 & 0.55 & 0.19 & 0.35 & 0.64 & 0.26 & 0.40 & 0.28 \\
\hline Unknown 11 & 1.50 & 1.02 & 0.68 & 8.60 & 3.05 & 0.35 & $0.000^{* *}$ \\
\hline Glucose & 0.76 & 0.51 & 0.67 & 4.18 & 1.71 & 0.41 & $0.000^{* *}$ \\
\hline Galactose & 0.80 & 0.35 & 0.44 & 1.19 & 0.22 & 0.18 & $0.001^{* *}$ \\
\hline Talose & 0.53 & 0.52 & 0.99 & 4.48 & 2.71 & 0.60 & $0.000^{* *}$ \\
\hline D-Gluconic acid & 0.11 & 0.13 & 1.11 & 0.16 & 0.05 & 0.30 & 0.26 \\
\hline Palmitic acid & 0.21 & 0.18 & 0.86 & 0.36 & 0.37 & 1.02 & 0.15 \\
\hline Inositol & 0.80 & 0.37 & 0.46 & 0.50 & 0.09 & 0.19 & $0.005^{* *}$ \\
\hline Ribose & 0.17 & 0.07 & 0.42 & 0.05 & 0.04 & 0.90 & $0.000^{* *}$ \\
\hline Mannitol & 0.21 & 0.19 & 0.87 & 0.27 & 0.08 & 0.31 & 0.34 \\
\hline Stearic acid & 0.07 & 0.04 & 0.59 & 0.13 & 0.14 & 1.11 & 0.10 \\
\hline Unknown 12 & 0.42 & 0.20 & 0.48 & 0.23 & 0.10 & 0.44 & $0.004^{* *}$ \\
\hline Unknown 13 & 0.70 & 0.39 & 0.56 & 0.38 & 0.11 & 0.29 & $0.006^{* *}$ \\
\hline
\end{tabular}

${ }^{1}$ Means, SD, and CV over all samples.

${ }^{2}$ Probability associated with the Student's $t$-test.

$* * P<0.01$. 
and pasteurized samples by an OPLS-DA. It resulted in a model with 1 predictive component and 1 orthogonal component and with $\mathrm{R}^{2}(\mathrm{X})=0.44, \mathrm{R}^{2}(\mathrm{Y})=0.83$, and $\mathrm{Q}^{2}(\mathrm{Y})=0.68$; the latter value indicated a higher uncertainty in class separation compared with that of the model concerning milk animal origin. We reported the score plot and the loading values in the top and bottom panel of Figure 3, respectively. Loading values indicated that U7 and U11, together with the sugars glucose and fructose had higher levels in UHT samples, whereas
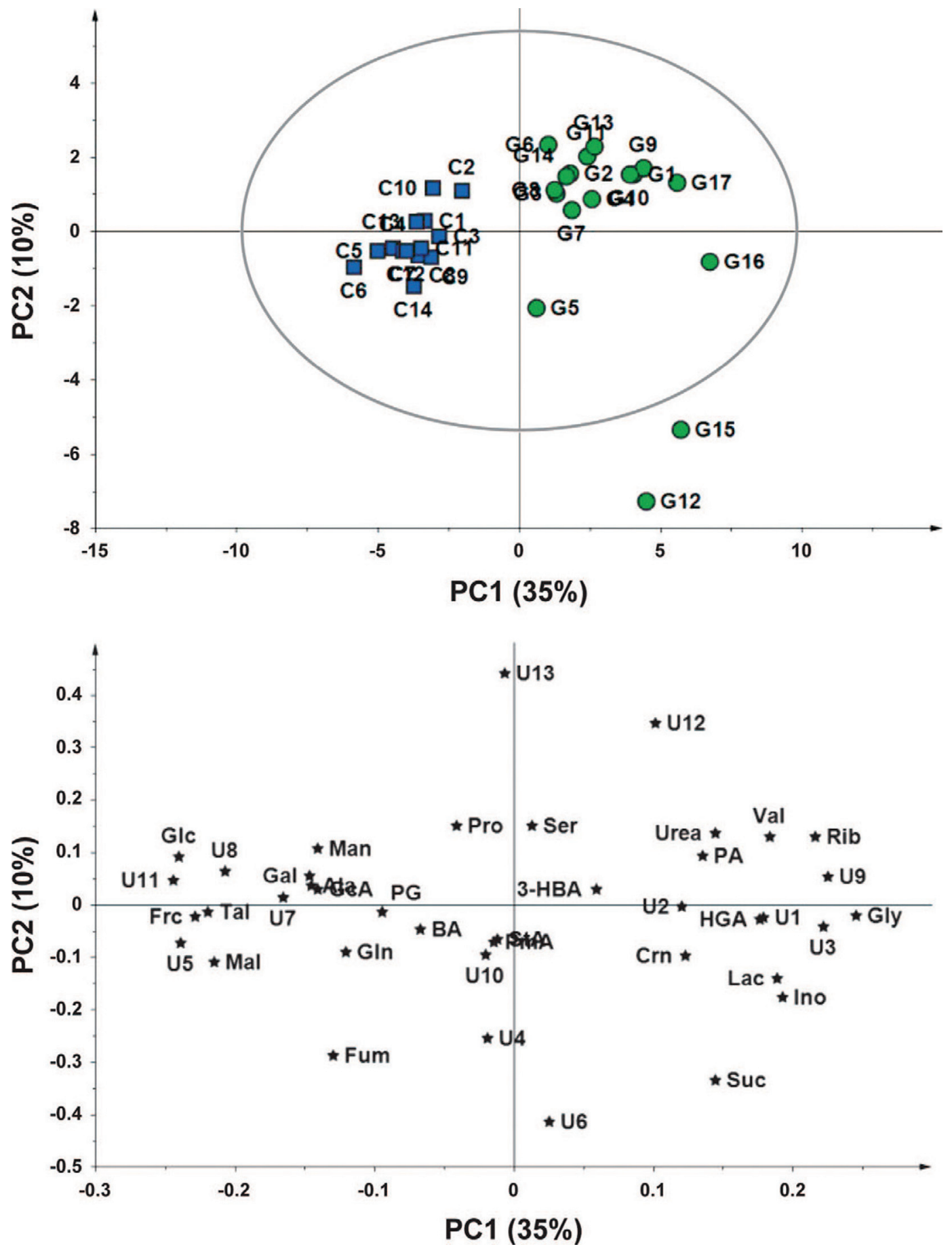

Figure 1. Principal components (PC) analysis of milk samples: PC1 versus PC2 score plot (top) of goat (G in green circles) and cow (C in blue squares) milk. The explained variance is reported in parentheses. The ellipse encloses the $95 \%$ Hotelling's $\mathrm{T}^{2}$ confidence region. The bottom panel shows the corresponding loading plot. Metabolites are abbreviated as proposed in Table 1. Color version available in the online PDF. 
hydroxyglutaric acid, U9, and ribose had higher levels in pasteurized samples. To target metabolites due solely to milk typology (goat vs. cow) and solely to heat treatments (UHT vs. pasteurization) and to avoid confounding overlapping, we projected the correlation loading vector of the predictive component in the goat and cow OPLS-DA model versus that of the OPLS-DA model concerning heat treatments. In such a way, we obtained the SUS plot reported in Figure 4. This 2-dimensional plot helped us to visualize both the shared
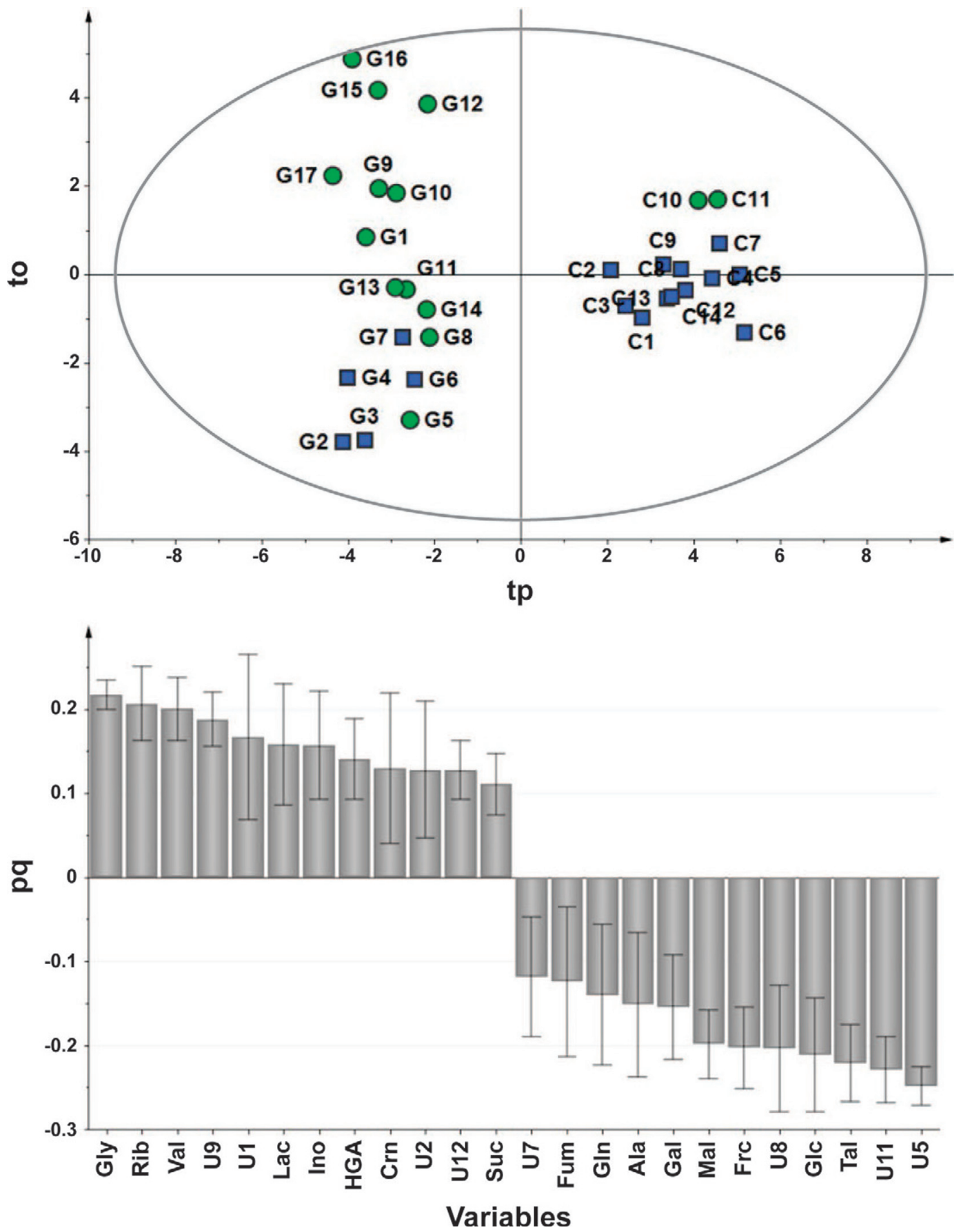

Figure 2. Orthogonal projections to latent structures-discriminant analysis of milk samples from different animal origin: score plot (top) of goat $(\mathrm{G})$ and cow milk $(\mathrm{C})$ samples, including pasteurized (green circles) and UHT-treated (blue squares) samples; tp = predictive component; to $=$ first orthogonal component. The bottom panel shows loading plot values along the predictive component, with error bars indicating the jackknifed confidence interval. Only metabolites with the highest loading values are reported and aligned in ascending order, with positive values for goat milk. Metabolites are abbreviated as proposed in Table 1. Color version available in the online PDF. 
and unique information of the 2 OPLS-DA models. The variables lined up along the diagonal running from the lower left corner to the upper right corner represented the shared structure between the 2 compared OPLS models. Conversely, variables that were not located along this diagonal represented structures that were unique to each class of the 2 compared models, always under the assumption that correlation loading vectors
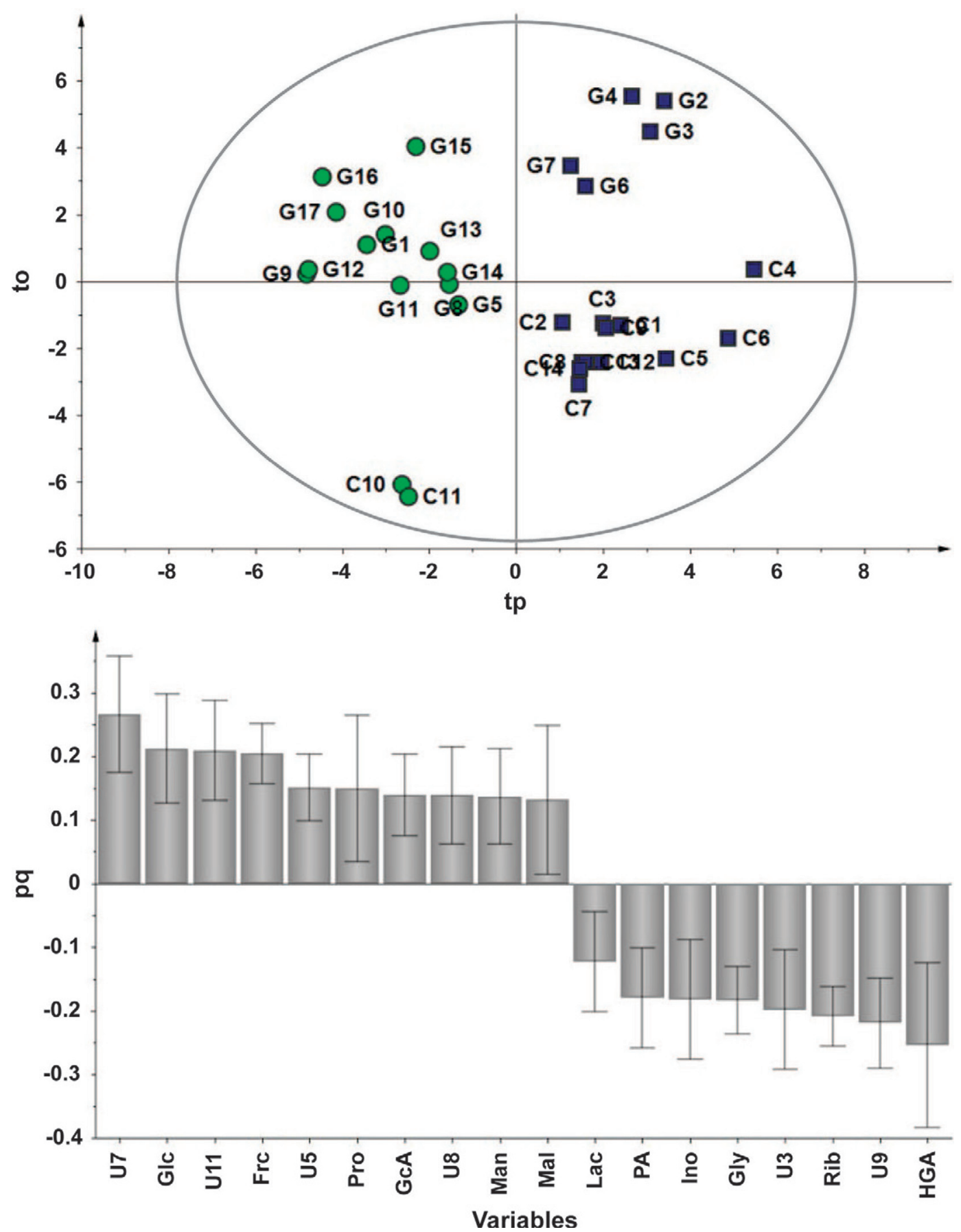

Figure 3. Orthogonal projections to latent structures-discriminant analysis of milk samples classified by heat treatments: score plot (top) of goat $(\mathrm{G})$ and cow milk (C) samples, including pasteurized (green circles) and UHT-treated (blue squares) samples; t1 = predictive component; to $=$ first orthogonal component. The bottom panel shows loading plot values along the predictive component, with error bars indicating the jackknifed confidence interval. Only metabolites with the highest loading values are reported and aligned in ascending order, with positive values for UHT milk. Metabolites are abbreviated as proposed in Table 1. Color version available in the online PDF. 
have significant values for the studied classes of samples. We observed that the metabolites with the highest correlations with goat milk were valine and glycine; it is worth recalling that valine, together with leucine and isoleucine, enters in metabolic pathways involved in the production of branched-chain FA, of which caprine milk is particularly rich (Massart-Leën and Massart, 1981). An important characteristic of goat milk is the unique flavor, attributed to differences in the fat fraction (Amigo and Fontecha, 2011); contribution to milk taste can be also given by valine (bitter) and glycine (sweet). The SUS plot also indicated that U5, talose, U8, and malic acid were the metabolites unique to bovine milk. The UHT samples resulted high in U7 and pasteurized samples resulted high in hydroxyglutaric acid (Figure 4). Ribose and U9 were shared by goat and pasteurized milk. Glucose, fructose, and U11 were shared by UHT and cow samples. This latter observation is in agreement with previous findings that indicate a higher presence of lactose in cow milk compared with goat milk (Amigo and Fontecha, 2011) and that, following UHT treatments, lactose is hydrolyzed to monosaccharidereducing sugars, which, reacting with amino groups, can give rise to undesired Amadori compounds (Mendoza et al., 2005). We also observed that other metabolites, although lower in correlation, exhibited interesting features; for example, proline exhibited a correlation value of approximately 0 for the goat versus cow OPLSDA model but had a correlation of 0.5 with UHT treatment class. In this regard, it has been reported that, in UHT milk, casein exhibits lower proline content (Tamime, 2009); a release of proline could then take place, thus increasing its presence as a free amino acid in the bulk.

The above-reported results proved that the GC-MSbased metabolomic approach was able to discriminate the 2 milk typologies (goat and cow) based on their metabolites. To test the predictive potentiality of the GC-MS profile for fraud detection, we submitted to MVA the analytical data of the prepared mixtures of goat and cow milk (see Materials and Methods section). A single-Y OPLS technique was used to construct the model that correlated the chromatographic data of the mixtures to the percentages of cow milk added as the $\mathbf{y}$ dependent variable. The obtained OPLS model had $\mathrm{R}^{2}(\mathrm{Y})=0.996$ and $\mathrm{Q}^{2}=0.879$; the accuracy for prediction in cross-validation of percentage of cow milk added, indicated by the root mean square error in cross-validation value, was approximately 5\%. A detectability of addition of cow milk as low as $5 \%$ can be considered a satisfactory result when such a fraud has to be detected; lower quantities are economically meaningless. This strategy can be proposed as a valid, fast, and cheap tool against commercial fraud regarding

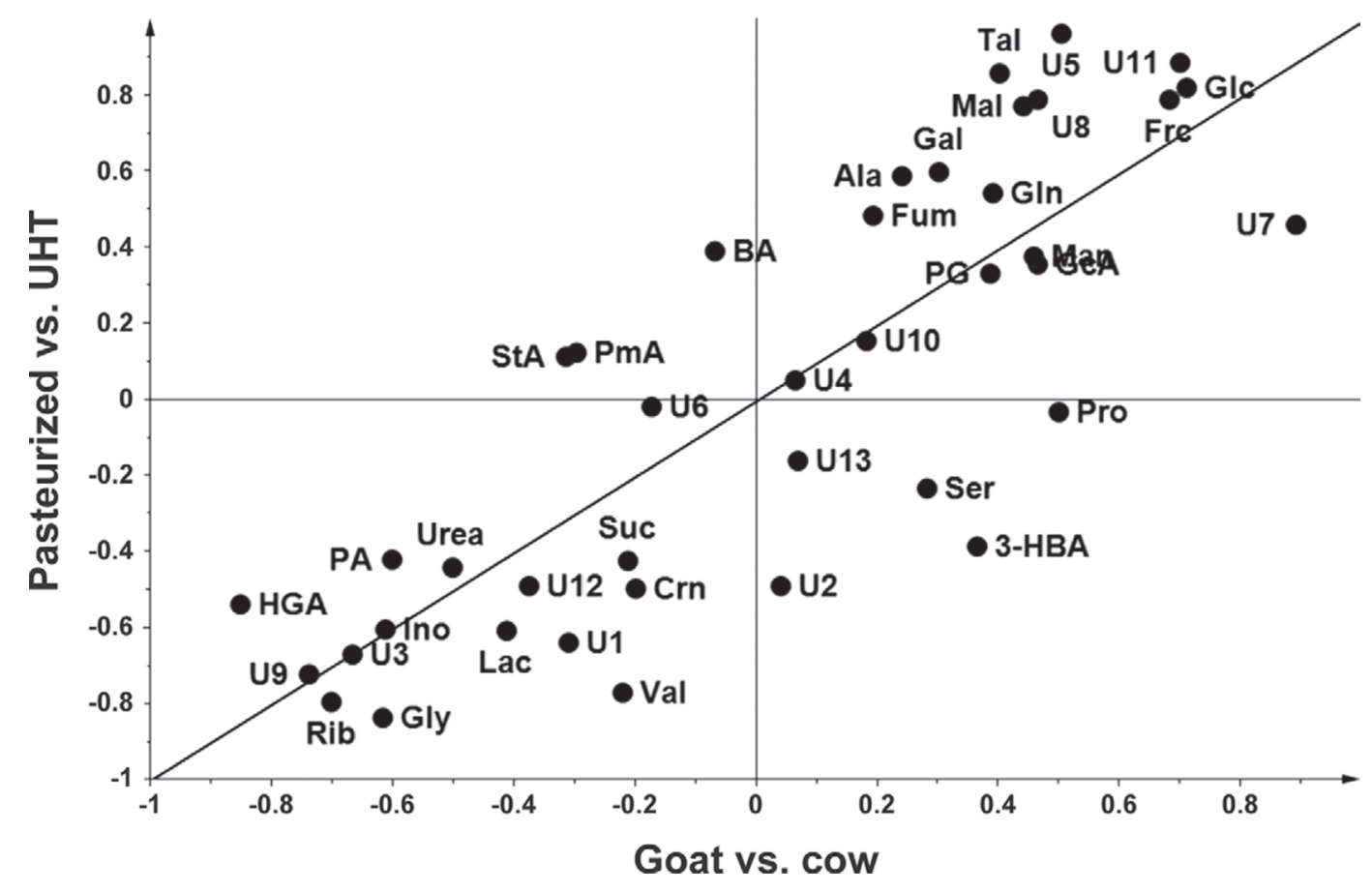

Figure 4. Shared and unique structures plot of the orthogonal projections to latent structures-discriminant analysis models of milk samples. The horizontal axis contains the loading correlation values of goat versus cow model and the vertical axis contains the loading correlation values of the pasteurized versus UHT model. Variables in the lower left corner are shared in goat and pasteurized samples; variables in the upper right corner are shared in cow and UHT samples. Metabolites are abbreviated as proposed in Table 1. 
mixtures of milk having different commercial values. As a final remark, we want to recall the extreme variability of goat milk reported by several authors (Haenlein, 2004; Amigo and Fontecha, 2011; Sabahelkheir et al., 2012); therefore, studies of caprine milk and derivatives could still be far from delineating standardizable results.

\section{ACKNOWLEDGMENTS}

This study was supported by grants provided by Regione Autonoma della Sardegna (Italy) "Valorizzazione e innovazione delle produzioni lattiero-casearie caprine in Sardegna. Indagini chimico-fisiche e nutrizionali" (Legge Regionale 7 Agosto 2007, N.7). The authors thank Ignazio Ibba of ARA Sardegna (Cagliari, Italy) for helpful suggestions.

\section{REFERENCES}

Amigo, L., and J. Fontecha. 2011. Milk | Goat milk. Pages 484-493 in Encyclopedia of Dairy Sciences. 2nd ed. J. W. Fuquay, P. F. Fox, and P. L. H. McSweeney, ed. Academic Press, Waltham, MA.

Antonilli, M., E. Bottari, and M. R. Festa. 2005. Ratios between content of fatty acids as markers for mixture of cow and goat milk. Ann. Chim. 95:501-514.

Boudonck, K. J., M. W. Mitchell, J. Wulff, and J. A. Ryals. 2009. Characterization of the biochemical variability of bovine milk using metabolomics. Metabolomics 5:375-386.

Ceballos, L. S., E. Ramos Morales, G. de la Torre Adarve, J. Díaz Castro, L. Pérez Martínez, and M. Remedios Sanz Sampelayo. 2009. Composition of goat and cow milk produced under similar conditions and analyzed by identical methodology. J. Food Compos. Anal. 22:322-329.

Chen, R.-K., L.-W. Chang, Y.-Y. Chung, M.-H. Lee, and Y.-C. Ling. 2004. Quantification of cow milk adulteration in goat milk using high-performance liquid chromatography with electrospray ionization mass spectrometry. Rapid Commun. Mass Spectrom. 18:1167-1171.

Dubeuf, J.-P., P. Morand-Fehr, and R. Rubino. 2004. Situation, changes and future of goat industry around the world. Small Rumin. Res. 2:165-173.

Eriksson, L., E. Johansson, N. Kettaneh-Wold, J. Trygg, C. Wikström, and S. Wold. 2013. Multi- and Megavariate Data Analysis. 3rd ed. Umetrics Academy, Umeå, Sweden.

Goetsch, A. L., S. S. Zeng, and T. A. Gipson. 2011. Factors affecting goat milk production and quality. Small Rumin. Res. 101:55-63.

Haenlein, G. F. W. 2004. Goat milk in human nutrition. Small Rumin. Res. 51:155-163.

Harzia, H., A. Ilves, M. Ots, M. Henno, I. Jõudu, T. Kaart, K. Ling, O. Kärt, K. Kilk, and U. Soomets. 2013. Alterations in milk metabolome and coagulation ability during the lactation of dairy cows. J. Dairy Sci. 96:6440-6448.
Hrbek, V., V. Vaclavik, O. Elich, and J. Hajslova. 2014. Authentication of milk and milk-based foods by direct analysis in real time ionization-high resolution mass spectrometry (DART-HRMS) technique: A critical assessment. J. Food Con. 36:138-145.

Klein, M. S., M. F. Almstetter, G. Schlamberger, N. Nürnberger, K. Dettmer, P. J. Oefner, H. H. D. Meyer, S. Wiedemann, and W. Gronwald. 2010. Nuclear magnetic resonance and mass spectrometry-based milk metabolomics in dairy cows during early and late lactation. J. Dairy Sci. 93:1539-1550.

Lamanna, R., A. Braca, E. Di Paolo, and G. Imparato. 2011. Identification of milk mixtures by ${ }^{1} \mathrm{H}$ NMR profiling. Magn. Reson. Chem. 49:S22-S26.

Levieux, D., and A. Venien. 1994. Rapid, sensitive two-site ELISA for detection of cows' milk in goats' or ewes' milk using monoclonal antibodies. J. Dairy Res. 61:91-99.

Locci, E. C. Piras, S. Mereu, F. C. Marincola, and P. Scano. 2011 ${ }^{1} \mathrm{H}$ NMR metabolite fingerprint and pattern recognition of mullet (Mugil cephalus) bottarga. J. Agric. Food Chem. 59:9497-9505.

Marincola, F. C, A. Noto, P. Caboni, A. Reali, L. Barberini, M. Lussu, F. Murgia, M. L. Santoru, L. Atzori, and V. Fanos. 2012. A metabolomic study of preterm human and formula milk by high resolution NMR and GC-MS analysis: Preliminary results. J. Matern. Fetal Neonatal Med. 25:62-67.

Massart-Leën, A. M., and D. L. Massart. 1981. The use of clustering techniques in the elucidation or confirmation of metabolic pathways. Application to the branched-chain fatty acids present in the milk fat of lactating goats. Biochem. J. 196:611-618.

Mendoza, M. R., A. Olano, and M. Villamiel. 2005. Chemical indicators of heat treatment in fortified and special milks. J. Agric. Food Chem. 53:2995-2999.

Raynal-Ljutovac, K., G. Lagriffoul, P. Paccard, I. Guillet, and Y. Chilliard. 2008. Composition of goat and sheep milk products: An update. Small Rumin. Res. 79:57-72.

Sabahelkheir, M. K., M. M. Fat en, and A. A. Hassan. 2012. Amino acid composition of human and animal's milk (camel, cow, sheep and goat). ARPN J. Sci. and Technol. 2:32-34.

Silanikove, N., G. Leitner, U. Merin, and C. G. Prosser. 2010. Recent advances in exploiting goat's milk: Quality, safety and production aspects. Small Rumin. Res. 89:110-124.

Sundekilde, U. K., L. B. Larsen, and H. C. Bertram. 2013. NMR-based milk metabolomics. Metabolites 3:204-222.

Tamime, A. Y., editor. 2009. Milk Processing and Quality Management. John Wiley \& Sons Ltd., Chichester, UK.

Troyano, E., M. Villamiel, A. Olano, J. Sanz, and I. Martinez-Castro. 1996. Monosaccharides and myo-inositol in commercial milks. J. Agric. Food Chem. 44:815-817.

Veselkov, K. A., L. K. Vingara, P. Masson, S. L. Robinette, E. Want, J. V. Li, R. H. Barton, C. Boursier-Neyret, B. Walther, T. M. Ebbels, I. Pelczer, E. Holmes, J. C. Lindon, and J. K. Nicholson. 2011. Optimized preprocessing of ultra-performance liquid chromatography/mass spectrometry urinary metabolic profiles for improved information recovery. Anal. Chem. 83:5864-5872.

Wiklund, S., E. Johansson, L. Sjöström, E. J. Mellerowicz, U. Edlund, J. P. Shockcor, J. Gottfries, T. Moritz, and J. Trygg. 2008. Visualization of GC/TOF-MS-based metabolomics data for identification of biochemically interesting compounds using OPLS class models. Anal. Chem. 80:115-122. 